\title{
Supporting Information: Evaporation-Induced Clogging of an Artificial Sweat Duct
}

\author{
Venkata Yashasvi Lolla, ${ }^{1, \dagger}$ Pranav Shukla, ${ }^{2, \dagger}$ Steven D. Jones,${ }^{3}$ and Jonathan B. \\ Boreyko $^{1, *}$ \\ ${ }^{1}$ Department of Mechanical Engineering, Virginia Tech, Blacksburg, VA 24061 \\ ${ }^{2}$ Department of Biology and Engineering Mechanics, Virginia Tech, Blacksburg, VA 24061 \\ 3 Procter \& Gamble, Mason, Cincinnati, OH 45040 \\ $\dagger$ Equal contribution \\ *To whom correspondence should be addressed; E-mail: boreyko@vt.edu.
}




\section{Contents}

1 Absorbency test for PDMS cubes $\quad$ S-3

\begin{tabular}{|lrl}
2 & Experimental results for critical pressure & S-4
\end{tabular}

\begin{tabular}{|lll}
3 & Determining diffusive vapor pressure of the sweat & S-5
\end{tabular}

$4 \quad$ List of ingredients and concentration of the artificial eccrine perspirant $\quad$ S-6

4.1 List of ingredients . . . . . . . . . . . . . . . . . . . . . S-6

4.1 .1 Metabolites . . . . . . . . . . . . . . . . . . S S-6

4.1 .2 Minerals $\ldots \ldots \ldots \ldots \ldots \ldots$. . . . . . . . . . . . . . . . .

4.1 .3 Amino acids . . . . . . . . . . . . . . . . S-6

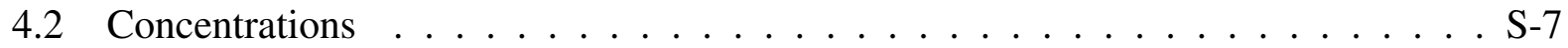

\begin{tabular}{lll}
\hline 5 & Video captions & S-8
\end{tabular} 


\section{Absorbency test for PDMS cubes}

The PDMS cubes were heated for $2 \mathrm{~h}$ at $80^{\circ} \mathrm{C}$ to get rid of any pre-existing moisture and were allowed to cool naturally. The initial mass of dry PDMS cubes was measured, after which each individual cube was dipped into a bath of water for a particular time period that ranged from $1 \mathrm{~s}$ to $1 \mathrm{~h}$. The PDMS cubes were then suspended for some time so as to remove any surplus water sticking to the cube. The weight of these cubes was measured again and the values were noted down for each cube. The water intake capacity for each cube was plotted against time. The capacity is quantified by the swelling ratio, defined as:

$$
\text { Swelling Ratio }=\frac{W_{\text {soaked }}-W_{\text {dry }}}{W_{\text {dry }}} \times 100,
$$

where $W_{\text {dry }}$ and $W_{\text {soaked }}$ are the weights of the PDMS before and after soaking, respectively. Irrespective of the soaking time period, almost all the cubes had a similar swelling ratio of about $1 \%$, showing that while PDMS does absorb some water, it was found to be mostly independent of the amount of time for which it was soaked.

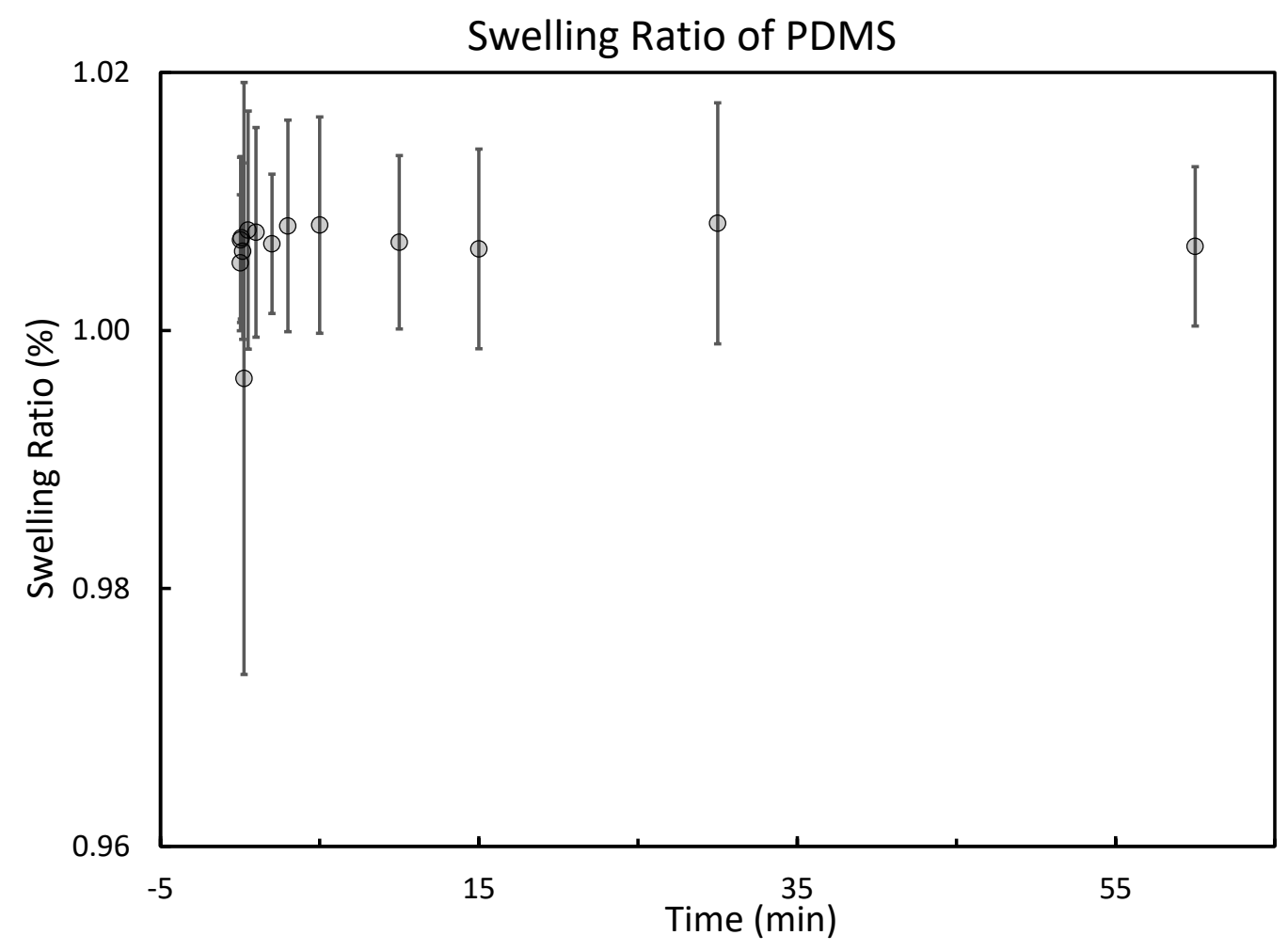

Figure S1: Swelling Ratio of PDMS. The x-axis represents the amount of time for which the PDMS cubes were dipped in the water bath. The error bars represent the standard deviation over three trials. 


\section{Experimental results for critical pressure}

Multiple experiments were performed to measure the critical pressure, $P_{\mathrm{c}}$, at which plug formation was observed. The distance between the tip of the microchannel and PDMS was maintained at $\delta=100 \mu \mathrm{m}$.

\begin{tabular}{|c|c|c|c|}
\hline Trial & $\delta(\mu \mathrm{m})$ & $\mathrm{PG}(\mathrm{g})$ & $\mathrm{P}_{\mathrm{c}}(\mathrm{psi})$ \\
\hline 1 & 100 & 0.0649 & 0.60 \\
2 & 100 & 0.1013 & 0.50 \\
3 & 100 & 0.1454 & 0.45 \\
4 & 100 & 0.0863 & 0.55 \\
5 & 100 & 0.0893 & 0.35 \\
6 & 100 & 0.0860 & 0.40 \\
7 & 100 & 0.0619 & 0.50 \\
8 & 100 & 0.0990 & 0.45 \\
9 & 100 & 0.0643 & 0.35 \\
\hline
\end{tabular}




\section{Determining diffusive vapor pressure of the sweat}
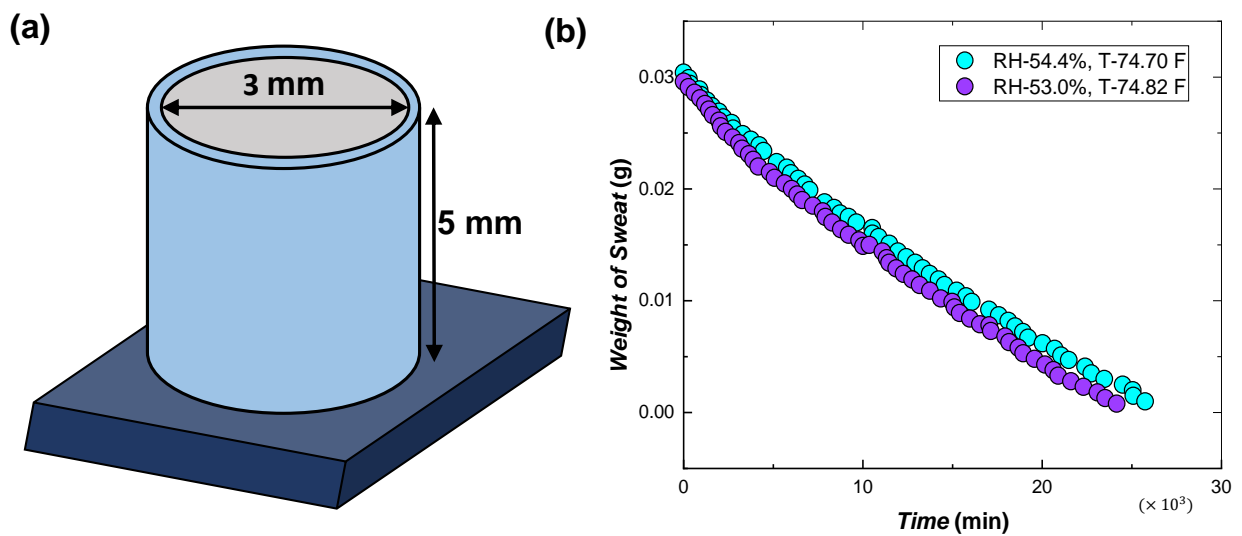

Figure S2: Diffusive evaporation of artificial sweat. (a) Schematic of a 3-D printed open cylinder containing artificial sweat. (b) Periodic recording of the mass of the sweat in the cylinder with respect to time for multiple trials.

The same artificial eccrine sweat used in the sweat duct experiments was poured into an open cylinder of height $5 \mathrm{~mm}$ and diameter $3 \mathrm{~mm}$. The change in the mass of the sweat due to diffusive evaporation was recorded periodically using a mass balance. The thickness of the concentration boundary layer, $\delta_{\mathrm{BL}}$, was estimated using the early-time mass flux and plugging it into the diffusion equation (Eq 4 in main manuscript). Holding the boundary layer constant over time, the change in vapor pressure of the sweat with increasing dehydration could then be extracted from Eq 4 using the evolving measurements in mass flux. 


\section{List of ingredients and concentration of the artificial eccrine perspirant}

The following list of ingredients and concentrations are obtained from Pickering Laboratories.

\subsection{List of ingredients}

\subsubsection{Metabolites}

- Uric Acid

- Urea

- Lactic Acid

- Ammonia

\subsubsection{Minerals}

- Sodium

- Zinc

- Chloride

- Calcium

- Copper

- Sulfate

- Magnesium

- Potassium

- Iron

- Nitrate

4.1.3 Amino acids

- Glycerine

- L-Alanine

- L-Arginine

- L-Asparagine

- L-Aspartic Acid 
- L-Citrulline

- L-Glutamic Acid

- L-Histidine

- L-Isoleucine

- L-Leucine

- L-Methionine

- L-Ornithine

- L-Phenylalanine

- L-Serine

- L-Threonine

- L-Tyrosine

- L-Valine

- Taurine

\subsection{Concentrations}

- Concentration for listed metabolites range from $0.007 \mathrm{~g} / \mathrm{L}$ (for Uric Acid) to $0.8 \mathrm{~g} / \mathrm{L}$ (for Urea).

- Concentration for listed minerals range from $0.001 \mathrm{~g} / \mathrm{L}$ (for Zinc) to $0.4 \mathrm{~g} / \mathrm{L}$ (for Calcium).

- Concentration for listed amino acids range from $0.001 \mathrm{~g} / \mathrm{L}$ (for Taurine) to $0.15 \mathrm{~g} / \mathrm{L}$ (for Serine). 


\section{Video captions}

Video 1: Represents the side view image of the video for three cases as described in the paper: (i) Flow of the sweat was studied near the tip of the microchannel when no PDMS cube was placed in front of the microchannel. A backflow was developed along the outer surface of the microchannel due to the formation of a stagnation point; (ii) A pure PDMS cube is now placed near the tip of the microchannel and its effect on the sweat was observed. No discernible features were found in this case that deviated from what was observed in case (i); (iii) A hygroscopic-infused PDMS cube was now introduced near the tip of the microchannel. Bubbling and shrinking of the backflow is first observed, eventually leading to formation of a gel like material that sticks to the outer surface of the microchannel. In the meantime, we see the sweat near the stagnation point receding into the microchannel. This eventually leads to complete clogging of the sweat due to formation of a plug via complete dehydration of the sweat inside the microchannel. 